\title{
Kinetics and Mechanism of Oligomer Desorption from Surfaces: $n$-Alkanes on Graphite
}

\author{
Andrew J. Gellman* and Kris R. Paserba \\ Department of Chemical Engineering, Carnegie Mellon University, Pittsburgh, Pennsylvania 15213
}

Received: May 7, 2002; In Final Form: October 3, 2002

\begin{abstract}
A previous study of the desorption of straight-chain alkanes $\left[\mathrm{H}\left(\mathrm{CH}_{2}\right)_{N} \mathrm{H}, N=5-60\right]$ from the surface of graphite has revealed that the measured desorption barriers, $\Delta E_{\mathrm{des}}^{\ddagger}$, have a nonlinear dependence on the chain length (Paserba, K. R.; Gellman, A. J. Phys. Rev. Lett. 2001, 86 (19), 4338). A model is proposed for the mechanism of oligomer desorption that accounts for the chain length dependence of the $\Delta E_{\mathrm{des}}^{\ddagger}$ through consideration of both the energy and entropy of oligomers interacting with a surface. The segments of the oligomer are identified with individual backbone bonds such that an alkane with $N$ carbon atoms has $I=N$ -1 segments. The oligomer segments are rapidly attaching to and detaching from the surface such that the various partially detached oligomers are in equilibrium with one another and can be classified by the number of detached segments $i$. Equilibrium among the partially detached oligomers is dictated by their relative free energies $\Delta A_{i}$, which are dependent on the numbers of detached segments. The energy $\mathrm{E}_{i}$ of an oligomer is simply linear in the number of detached segments. The entropy, $\mathrm{S}_{i}$, is given by the statistics for detachment of $i$ of $I$ segments from the surface and by the number of trans-gauche conformations about each detached $\mathrm{C}-\mathrm{C}$ bond. Finally, desorption is irreversible and occurs via a transition state with all segments detached from the surface. This model has been used to derive an expression for the measured $\Delta E_{\mathrm{des}}^{\ddagger}$ which accounts for the contributions of both segment-surface detachment and trans-gauche conformational isomerism. The theory accurately reproduces the nonlinear dependence of the $\Delta E_{\mathrm{des}}^{\ddagger}$ on chain length for alkane desorption from graphite.
\end{abstract}

\section{Introduction}

The interactions of polymers or shorter chain oligomeric species with surfaces plays an important role in a number of physically and technologically important processes. As such, understanding the properties of adsorbed oligomers is an important and fundamental problem. The particular example that has motivated the work described in this paper is that of the evaporation of perfluoropolyalkyl ether (PFPE) lubricants from the surfaces of hard disk data storage media. Hard disks are coated with a 50-100 ̊ overcoat of amorphous hydrogenated carbon $\left(\mathrm{a}-\mathrm{CH}_{x}\right)$ which is lubricated with a thin film of PFPE lubricant. ${ }^{1-3}$ The PFPEs are oligomers with backbone chain lengths of 50-150 atoms and are deposited as films of 10-20 $\AA$ thickness. Evaporation is an important mechanism of lubricant loss from the disk surface over the course of its life. Since these films are only 1-2 monolayers thick this is a problem in oligomer desorption from surfaces. Experimentally, we have modeled this problem by studying the desorption kinetics of straight-chain alkanes and straight-chain poly(ethylene glycol)s from the surface of graphite. The principle finding of these studies is that the desorption barriers, $\Delta E_{\text {des }}^{+}$, that one measures using temperature programmed desorption (TPD) measurements are nonlinear in the chain length. This paper describes a model for the oligomer-surface interaction and for the mechanism of alkane desorption from graphite that quantitatively reproduces this nonlinear dependence of $\Delta E_{\mathrm{des}}^{\ddagger}$ on chain length.

Temperature programmed desorption of small molecules from surfaces is one of the most common experiments in surface chemistry and is the simplest approach to measuring the $\Delta E_{\mathrm{des}}^{\ddagger}$

* To whom correspondence should be addressed. E-mail: ag4b@andrew.cmu.ed. of molecules that are reversibly adsorbed on surfaces in a vacuum. The desorption process is usually understood in fairly simple terms as translation of the molecule along the surface normal from its adsorbed state to a desorbed state. In this case the reaction coordinate is simply the surface normal and one describes the process with a simple one-dimensional potential energy surface. The first-order rate constant for desorption is described with transition state theory as

$$
k_{\mathrm{des}}=\left(\frac{k_{\mathrm{B}} T}{h}\right) \frac{q_{\ddagger}}{q} \exp \left(\frac{-\Delta E_{\mathrm{des}}^{\ddagger}}{k_{\mathrm{B}} T}\right)
$$

where $q_{\#}$ is the partition function for the transition state to desorption, $q$ is the partition function for the adsorbed molecule and $\Delta E_{\mathrm{des}}^{*}$ is the difference in the zero-point energies of the adsorbed molecule and the transition state to desorption. Under the assumption that the adsorption process is nonactivated, the value of $\Delta E_{\mathrm{des}}^{\ddagger}$ is often believed to approximate the desorption energy $\Delta E_{\text {des. }}$. Although small molecules always have degrees of freedom other than the reaction coordinate for desorption, those other degrees of freedom are only considered to influence desorption kinetics through the partition functions that appear in the rate constant $k_{\text {des. }}$. They do not significantly influence the measured value of the $\Delta E_{\mathrm{des}}^{\ddagger}$ for small molecules desorbing from surfaces.

It is easy to imagine that the complex and loose structure of oligomeric chains may influence the dynamics of their behavior on surfaces and the kinetics of their desorption from surfaces. In the case of alkanes on graphite there are numerous STM images showing that at room temperature they adopt an alltrans configuration with their molecular axis and molecular plane parallel to the surface. ${ }^{4-10}$ In principle, desorption could occur 
by the simultaneous translation of all segments away from the surface along the surface normal. Under these circumstances one would expect the $\Delta E_{\mathrm{des}}^{\ddagger}$ to be linear in the chain length. Alternatively, one could imagine a multitude of more complex but energetically equivalent pathways involving random, independent detachment of the segments from the surface ultimately leading to desorption. Statistically, such a mechanism seems more likely than the simultaneous, concerted translation of all segments away from the surface. Although prior experiments with alkanes and alcohols have been interpreted to suggest that the $\Delta E_{\mathrm{des}}^{\ddagger}$ is linear in chain length those studies have all been restricted to chains of 12 atoms or less. ${ }^{1-18}$ The effects of multiple desorption pathways should become more prominent as the chain length increases. This is quite apparent in the work presented in this paper and leads to a nonlinear dependence of the measured $\Delta E_{\text {des }}^{\ddagger}$ on chain length. ${ }^{19,20}$

The prior relevant studies of adsorbed oligomers on surfaces can be divided into two types: those that have measured the $\Delta E_{\mathrm{des}}^{\ddagger}$ for short chain oligomers adsorbed on surfaces in a vacuum ${ }^{11-14}$ and those that have used scanning tunneling microscopy (STM) to image the structures of longer chain oligomers adsorbed on surfaces. ${ }^{4-10}$ As mentioned there have been several previous studies which have investigated the effects of chain length on the desorption kinetics of alkyl alcohols and simple alkanes adsorbed on various surfaces. Zhang and Gellman used TPD to study the reversible adsorption of a series of straight-chain alcohols $\left(\mathrm{CH}_{3}\left(\mathrm{CH}_{2}\right)_{N-1} \mathrm{OH}, N=1-5\right)$ on the $\mathrm{Ag}(110)$ surface and concluded that the $\Delta E_{\text {des }}^{\ddagger}(N)$ increased incrementally by $4.6 \pm 0.4 \mathrm{~kJ} / \mathrm{mol}$ per methylene group in the hydrocarbon chain. ${ }^{14}$ Millot et al. investigated the desorption of $n$-alkanes $(n=4-8)$ from silicalite crystals using TPD and found that the $\Delta E_{\mathrm{des}}^{\ddagger}(N)$ also scaled linearly with chain length but in increments of $13.5 \mathrm{~kJ} / \mathrm{mol}$ per methylene unit. ${ }^{13}$ The desorption of $n$-alkanes $(n=6-12)$ from the $\mathrm{Au}(111)$ surface was studied by Wetterer et al. using helium atom reflectivity. ${ }^{12}$ Their conclusion from that study was that the $\Delta E_{\mathrm{des}}^{*}(N)$ increased incrementally by $6.2 \pm 0.2 \mathrm{~kJ} / \mathrm{mol}$ per methylene unit. Finally, a recent study by Bishop et al. has reported segmentsurface interaction energies of $\sim 8 \mathrm{~kJ} / \mathrm{mol}$ per methylene group for $n$-alkanes $(N=6-10)$ adsorbed on $\mathrm{Pt}(111){ }^{11}$ This was the first study with data suggesting that the $\Delta E_{\mathrm{des}}^{*}(N)$ may not be truly linear but the limited range of the chain lengths makes it difficult to determine the functional form of this nonlinearity. In these cases the range of alkyl chain lengths has been limited to $N \leq 12$. Needless to say, it is not surprising that over the limited range of chain lengths used in these studies the $\Delta E_{\text {des }}^{\ddagger}(N)$ would appear to be linear.

The structure of a number of oligomers adsorbed on surfaces has been studied through the use of STM. The majority of the STM images have shown that at room temperature the alkanes adopt all-trans configurations with their molecular planes lying parallel to the surface. ${ }^{4-10}$ Askadskaya and Rabe used a variable temperature STM to image monolayer coverages of $\mathrm{C}_{24} \mathrm{H}_{50}$ and $\mathrm{C}_{32} \mathrm{H}_{66}$ on graphite in air. ${ }^{6}$ High-resolution images of each monolayer were obtained at 297, 303, 313, and 318 K. At 297 $\mathrm{K}$, highly ordered lamellae of the $n$-alkanes were observed with their carbon atoms in an all-trans conformation and their molecular backbone parallel to the surface. Heating to $303 \mathrm{~K}$ induced a small dynamic roughening of the lamellae. Further heating to $313 \mathrm{~K}$ caused a continuing increase in roughness, however, the spacing between the molecules within the lamella remained essentially constant. Finally, at $318 \mathrm{~K}$ no molecular structure was observed for the $n$-alkanes on graphite. Upon cooling, the lamellae assumed their original structures, indicating the reversible nature of the disordering observed with the STM. The fundamentally important result of that work is that substrate temperature will affect the conformations of adsorbed oligomers by causing deviations from the all-trans configurations observed at low temperatures. Thermally induced disorder and conformational defects in $n$-alkane monolayers on graphite have also been investigated by Bucher et al. ${ }^{5}$ STM images were recorded for $\mathrm{C}_{28} \mathrm{H}_{58}$ and $\mathrm{C}_{32} \mathrm{H}_{66}$ at 281 and $300 \mathrm{~K}$, respectively. At 281 $\mathrm{K}$ the monolayers of each molecule formed lamellar structures with the molecules in an all-trans conformation, as previously reported. In an effort to investigate the thermal disordering of these monolayers, Bucher et al. utilized a quench technique whereby the graphite substrate was heated to a temperature near the bulk melting point of the adsorbed $n$-alkane and then rapidly cooled at a rate of $200 \mathrm{~K} / \mathrm{min}$ to a final temperature of $283 \mathrm{~K}$. This rapid quenching allowed "frozen-in" structures of the hightemperature phase to be imaged using the STM. During these experiments, the monolayers of $\mathrm{C}_{28} \mathrm{H}_{58}$ and $\mathrm{C}_{32} \mathrm{H}_{66}$ on the graphite surface were heated to 346 and $342 \mathrm{~K}$, respectively. The choice of temperatures was made in order to allow study of the conformational disorder that occurs at temperatures slightly below $\left(\mathrm{C}_{32} \mathrm{H}_{66}\right)$ and slightly above $\left(\mathrm{C}_{28} \mathrm{H}_{58}\right)$ the bulk melting points of the $n$-alkanes. After quenching, STM images were recorded for $\mathrm{C}_{28} \mathrm{H}_{58}$ and $\mathrm{C}_{32} \mathrm{H}_{66}$ at 279 and $287 \mathrm{~K}$, respectively. In each case, the STM images revealed a significant loss of molecular order. The identity of the individual lamellae present in the low temperature equilibrium structure was completely lost in the quenched films as a result of conformational disorder. These phenomena are similar to those observed by Askadskaya and Rabe in their studies. ${ }^{6}$ All the variable temperature STM images of long chain $n$-alkanes adsorbed on graphite suggest that heating causes displacement of methylene groups from the surface and may thereby decrease the $\Delta E_{\mathrm{des}}^{\ddagger}$ relative to that expected for $n$-alkanes in an all-trans conformation.

The work described in this paper has gone far beyond the previous studies of oligomer desorption in that the range of alkanes used, $\mathrm{C}_{5} \mathrm{H}_{12}$ to $\mathrm{C}_{60} \mathrm{H}_{122}$, is far more extensive. ${ }^{19,21}$ These were adsorbed on graphite in ultrahigh vacuum and then desorbed by heating the graphite at a constant rate. Desorption rates were measured using mass spectrometry. The results of these experiments have been published previously and clearly show that over this range of oligomer chain lengths the measured values of $\Delta E_{\mathrm{des}}^{\ddagger}(N)$ are nonlinear in chain length $N$ (Figure 1). Empirically it was shown that the desorption barriers could be fit to a power law of the form $\Delta E_{\mathrm{des}}^{*}(N)=a+b N^{\gamma}$, with the exponent having a value of $\gamma=0.50 \pm 0.01$. Since then further experimental work has examined the desorption kinetics of poly(ethylene glycol)s $\left[\mathrm{HO}\left(\mathrm{CH}_{2} \mathrm{CH}_{2} \mathrm{O}\right)_{n} \mathrm{H}\right]$ and poly(ethylene glycol) dimethyl ethers $\left[\mathrm{CH}_{3} \mathrm{O}\left(\mathrm{CH}_{2} \mathrm{CH}_{2} \mathrm{O}\right)_{n} \mathrm{CH}_{3}\right]$ with chain lengths covering a range similar to that used in the study of the alkanes. $^{22}$ The chain length dependence of the $\Delta E_{\mathrm{des}}^{\ddagger}$ for poly(ethylene glycol) dimethyl ethers adsorbed on graphite is almost identical to that observed for the alkanes. The chain length dependence of the $\Delta E_{\mathrm{des}}^{\ddagger}$ for poly(ethylene glycol)s adsorbed on graphite is also nonlinear but appears to reveal the effects of the functional endgroups for short chain lengths. The fact is that the nonlinear dependence of the $\Delta E_{\mathrm{des}}^{\ddagger}$ on chain length has been observed in all these measurements and appears to be a general phenomenon.

Our previous description of the desorption kinetics of alkanes from the graphite surface included an initial model for the desorption process and for the dynamics of the alkanes on the graphite surface. ${ }^{20}$ This model will be described in more detail 


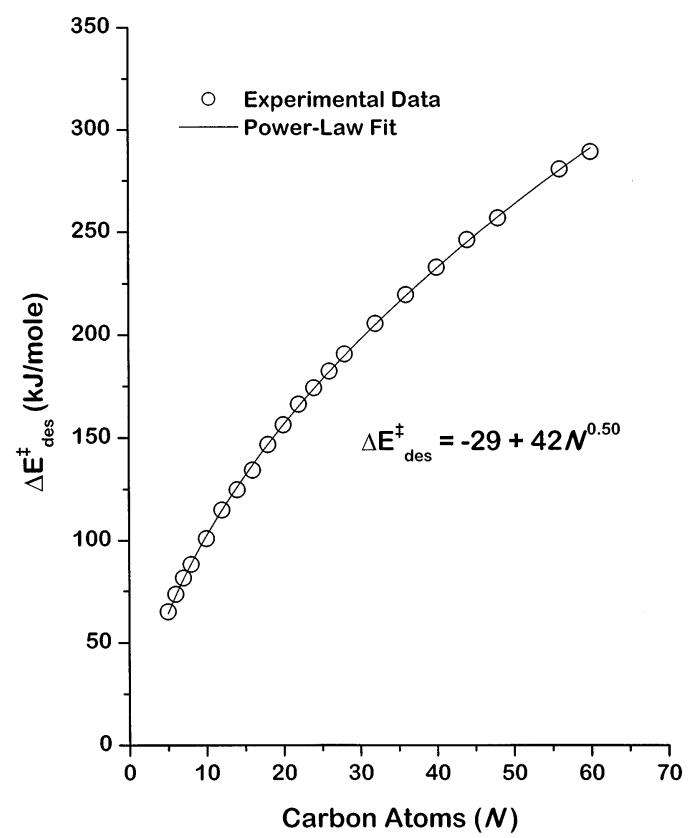

Figure 1. The values of $\Delta E_{\mathrm{des}}^{\ddagger}$ for alkanes on graphite measured as a function of the chain length, $N$. These illustrate the nonlinear dependence of $\Delta E_{\mathrm{des}}^{*}$ on $N$. The solid line is a fit of the data to the empirical form $\Delta E_{\mathrm{des}}^{\ddagger}(N)=a+b N^{\gamma}$.

later in this paper but, briefly, the idea is that desorption can be described as a process in which the individual segments of the oligomer are attaching and detaching from the surface rapidly such that the partially detached alkanes are in equilibrium with one another. The partially detached states of a molecule with $I$ segments are described by the number of detached segments $i$. Describing the equilibrium between the partially detached oligomers relies on the use of expressions for their energies $E_{i}$, entropies $S_{i}$, and free energies $\left(A_{i}=E_{i}-\mathrm{TS}_{i}\right)$. The energy is taken to be linear in the number of detached segments $i$. The entropy is determined by the number of ways of detaching $i$ of $I$ segments from the surface and by the number of trans - gauche conformations that can be adopted by the detached $\mathrm{C}-\mathrm{C}$ bonds. Finally, irreversible desorption occurs via a transition state with all segments detached from the surface and can be described using transition state theory. This allows one to derive an analytical expression for the $\Delta E_{\mathrm{des}}^{\ddagger}$ measured for oligomers of different length. This expression can be used to fit the experimental values of $\Delta E_{\mathrm{des}}^{\ddagger}(N)$ extremely well using only two free parameters: a segment-surface interaction energy, $\Delta E^{\mathrm{CC}}$, and a barrier to desorption of the fully detached oligomer from the surface, $\Delta E$.

This paper focuses on oligomer desorption and the dynamics of oligomers adsorbed on surfaces and makes a number of substantial improvements to the initial attempt to model these phenomena. One of the assumptions originally used to simplify the expression for $\Delta E_{\mathrm{des}}^{\ddagger}(N)$ has been eliminated allowing us to derive a rigorous, analytical expression for $\Delta E_{\mathrm{des}}^{\ddagger}(N)$. Another improvement to our model is the explicit inclusion of the temperature dependence of the trans-gauche conformations of the alkanes about their $\mathrm{C}-\mathrm{C}$ bonds. Originally, it was assumed simply that each detached $\mathrm{C}-\mathrm{C}$ bond could adopt three configurations: trans, +gauche, and -gauche. In reality there is some energy difference, $\Delta E_{t g}^{\mathrm{CC}}$, between the trans and the gauche configurations and this imparts a temperature dependence to the partition function for the trans-gauche conformations about $\mathrm{C}-\mathrm{C}$ bonds. As it turns out this has a significant impact on the values of $\Delta E_{\mathrm{des}}^{\ddagger}(N)$. Finally, the results have been used

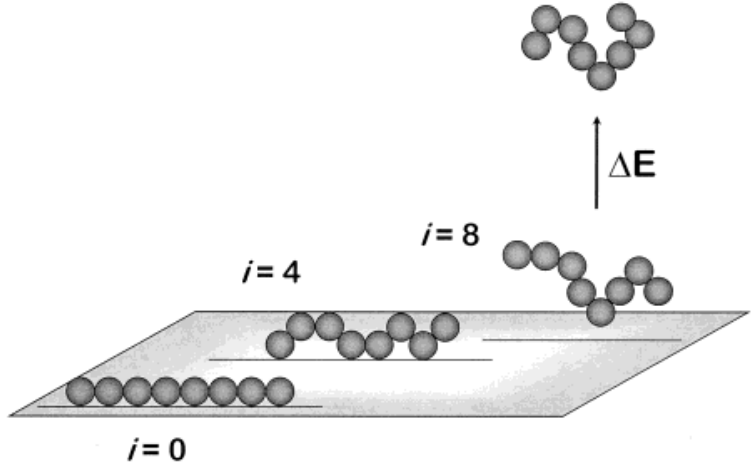

Figure 2. A schematic illustration of the process of oligomer detachment from a surface. In this figure the spheres represent oligomer segments which in this work are equated with $\mathrm{CC}$ bonds in the adsorbed alkanes. In this model the alkane with $N$ carbon atoms has $I=N-1$ segments. The index $i$ is used to denote the number of detached segments. The oligomer evolves from the fully attached state with $i=$ 0 through to the state with all segments detached from the surface, $i=$ I. All these steps are reversible and thus all partially detached species are in equilibrium with one another. The irreversible step is the desorption of the fully detached species.

to predict the average configuration of alkanes adsorbed on surfaces at elevated temperatures.

\section{The Oligomer Desorption Process}

We will begin initially by discussing the desorption of a homopolymer or oligomer in which all segments are identical. For the purposes of this work the number of segments in an oligomer is equated with the number of bonds. In this case an alkane with $N$ carbon atoms then has $I=N-1$ segments or $\mathrm{C}-\mathrm{C}$ bonds. Thinking of the bonds rather than the atoms as segments makes it easy to enumerate the number of transgauche conformations that can be adopted by oligomers with a given number of segments detached from the surface.

As mentioned above the segments of the adsorbed oligomers are considered to be rapidly detaching from and reattaching to the surface. The detachment of a segment is an activated process which would require some energy $\Delta E^{\mathrm{CC}}$. By comparison with detachment, the reattachment of a segment to the surface should be much more rapid since it should not be activated. Reattachment may, however, involve some entropic penalty since the detached $\mathrm{C}-\mathrm{C}$ bonds can adopt multiple conformations while the attached bonds are restricted to the trans configuration. The detachment-attachment kinetics of individual segments should be much more rapid than the net rate of oligomer desorption so it should be appropriate to think of the partially detached oligomers as being in equilibrium with one another. For the purposes of this work the partially detached states of an oligomer with $I$ segments will be enumerated simply by the number of detached segments, $i$. Figure 2 shows a schematic of the detachment and desorption process for an oligomer with 8 segments or $\mathrm{C}-\mathrm{C}$ bonds. Note that each of the spherical segments in the oligomer shown in Figure 2 represents a $\mathrm{C}-\mathrm{C}$ bond rather than a methylene group. The partially detached species are in equilibrium with one another on the surface. Desorption of the oligomer from the surface is an irreversible process. Once the final segment is detached the oligomer desorbs with some energy barrier, $\Delta E$.

\section{Rate Constant for Oligomer Desorption}

If the coverages of the partially detached oligomers are given by $\theta_{i}$, then the rate of oligomer desorption is given in terms of 
the coverage $\theta_{I}$ of oligomers with all segments detached from the surface as

$$
r=\left(\frac{k_{\mathrm{B}} \mathrm{T}}{h}\right) \frac{q_{\ddagger}}{q_{I}} \exp \left(\frac{-\Delta E}{k_{\mathrm{B}} T}\right) \theta_{I}
$$

The partition functions are for the species with all segments detached, $q_{I}$, and for the transition state to desorption, $q_{\ddagger}$. Unfortunately, the form of the rate expression above is not of direct use since one cannot know or control the coverage, $\theta_{I}$, of species with all segments detached from the surface. As a result we are faced with the problem of relating the coverage $\theta_{I}$ to the total coverage of adsorbed oligomers, $\theta$.

Finding an expression for the rate of desorption in terms of the total coverage of adsorbed oligomers is made possible by the fact that all the partially detached oligomers are in equilibrium with one another. Equilibrium implies that the relative coverages of partially detached oligomers are all dictated by equilibrium constants. The coverages of all species can be related to the coverage $\theta_{0}$ of the fully attached oligomer through the equilibrium constants $\mathrm{K}_{i}$ and

$$
\theta_{i}=K_{i} \theta_{0}=\exp \left(\frac{-\Delta A_{i}}{k_{\mathrm{B}} T}\right) \theta_{0}
$$

The free energy difference, $\Delta A_{i}$, in this expression for the equilibrium constant is just given by the difference in free energies of the oligomer with $i$ detached segments and the fully attached oligomer.

$$
\Delta A_{i}=A_{i}-A_{0}
$$

The total coverage $\theta$ of the oligomers is given by the sum over the coverages of oligomers with all possible numbers of detached segments.

$$
\begin{aligned}
\theta & =\sum_{i=0}^{I} \theta_{i} \\
& =\theta_{0} \sum_{i=0}^{I} \exp \left(\frac{-\Delta A_{i}}{k_{\mathrm{B}} T}\right) \\
& =\Gamma \theta_{0}
\end{aligned}
$$

Now the coverage of oligomers with all segments detached is given by

$$
\begin{aligned}
\theta_{I} & =\exp \left(\frac{-\Delta A_{I}}{k_{\mathrm{B}} T}\right) \theta_{0} \\
& =\frac{1}{\Gamma} \exp \left(\frac{-\Delta A_{I}}{k_{\mathrm{B}} T}\right) \theta
\end{aligned}
$$

where

$$
\Gamma=\sum_{i=0}^{I} \exp \left(\frac{-\Delta A_{i}}{k_{\mathrm{B}} T}\right)
$$

Finally, the rate of oligomer desorption can be written as

$$
r=\left(\frac{k_{\mathrm{B}} T}{h}\right) \frac{q_{\ddagger}}{q_{I} \Gamma} \exp \left(\frac{-\Delta A_{I}}{k_{\mathrm{B}} T}\right) \exp \left(\frac{-\Delta E}{k_{\mathrm{B}} T}\right) \theta
$$

This rate expression is now in a useful form in the sense that it is written in terms of the total oligomer coverage $\theta$, a variable that can be controlled or measured experimentally.

The rate expression above defines the first-order rate constant for oligomer desorption from a surface.

$$
\begin{aligned}
k_{\mathrm{des}} & =\left(\frac{k_{\mathrm{B}} T}{h}\right) \frac{q_{\ddagger}}{q_{I} \Gamma} \exp \left(\frac{-\Delta A_{I}}{k_{\mathrm{B}} T}\right) \exp \left(\frac{-\Delta E}{k_{\mathrm{B}} T}\right) \\
& =\left(\frac{k_{\mathrm{B}} T}{h}\right) \frac{q_{\ddagger}}{\Gamma} \exp \left(\frac{-\left(\Delta E_{I}+\Delta E\right)}{k_{\mathrm{B}} T}\right)
\end{aligned}
$$

To evaluate this expression for a given oligomer one needs expressions for the energies $E_{i}$, the entropies $S_{i}$, and thus the free energies, $A_{i}$, of the oligomers in all their stages of partial detachment. Given expressions for the energy and the entropy of the partially detached oligomers, the experimentally measured desorption barrier can be found by evaluating

$$
\Delta E_{\mathrm{des}}^{\ddagger}=-k_{\mathrm{B}} \frac{\mathrm{d} \ln \left(k_{\mathrm{des}}\right)}{\mathrm{d}(1 / T)} .
$$

Given the nature of the desorption process as described in section 2 one may be inclined to argue that there are many desorption channels that should be considered rather than just the desorption of oligomers with all segments detached from the surface. For example one may suggest that rate constants should be considered for those species with one, two, or more segments still attached to the surface. The original description of the desorption process in the text of ref 20 is a little misleading in this regard in that it suggests that the only species that can desorb at any instant in time are those with only one segment left attached to the surface. The model is quantified, however, using transition state theory, and it is important to keep in mind the fact that within the context of transition state theory the adsorbed oligomers are all in equilibrium with one another and with the transition state to desorption. As such there are no independent desorption channels or paths to be considered. The equilibrium is path independent and the point of this work is not to describe a mechanistic pathway but instead to explicitly account for the equilibrium among the adsorbed oligomers and its influence on the measured values of $\Delta E_{\mathrm{des}}^{\ddagger}(N)$. Another way of articulating this is to point out that the rate constant given by eq 9 is exactly what one would expect from transition state theory for oligomer desorption. The exponential term simply contains the energy difference between the transition state and the minimum energy or fully attached state. The quantity $q_{\ddagger}$ is the partition function for the transition state. The quantity $\Gamma$ is simply the partition function for the adsorbed oligomer but it is important to note that it includes contributions from all the partially detached states which are in equilibrium with one another and with the transition state.

\section{Oligomer-Surface Interaction}

Given the expression in eq 9 for the desorption rate constant, we now need a model for the oligomer-surface interactions that will yield the energies and entropies of the partially detached oligomers. For our purposes the reference energy state will be the fully adsorbed oligomer with $i=0$ detached segments and $E_{0}=0$. The simplest expression for the energy of a partially detached oligomer is one that is linear in the number of detached segments.

$$
E_{i}=i \Delta E^{\mathrm{CC}}
$$


This defines an unknown parameter the segment-surface interaction energy $\Delta E^{\mathrm{CC}}$. One could also imagine a more complex model for the oligomer-surface attractions which takes account of possible interactions between detached segments. As will be shown later the inclusion of such interactions does not have a significant impact on $\Delta E_{\mathrm{des}}^{\ddagger}(N)$ for oligomers in the length range considered here.

The entropy of the partially detached oligomers is determined by their partition functions through the Boltzmann equation. Roughly speaking, the partition functions can be divided into a configurational term, $q_{i}^{\mathrm{c}}$, arising from the number of configurations that the oligomers can adopt and a second term, $q_{i}^{\text {vtr }}$, that describes the contributions from other degrees of freedom such as vibration, translation, and rotation. Separated in this way the partition function for an oligomer with $i$ segments detached from the surface is given by

$$
q_{i}=q_{i}^{\mathrm{vtr}} q_{i}^{\mathrm{c}}
$$

Most of the degrees of freedom that contribute to $q_{i}^{\mathrm{vtr}}$ are present in both the adsorbed state and in the transition state to desorption and thus their partition functions roughly cancel in the rate constant given by transition state theory. Furthermore, the $q_{i}^{\text {vtr }}$ are only weakly temperature dependent and thus do not contribute greatly to the measured values of $\Delta E_{\mathrm{des}}^{\ddagger}$. The possible exception is translational freedom parallel to the surface which may exist in the transition state but not in the adsorbed state. This could contribute to the preexponent for desorption but since the partition function for translation in the two degrees of freedom parallel to the surface is linear in temperature it would not contribute significantly to the measure value of $\Delta E_{\mathrm{des}}^{\ddagger}$. In contrast with the translational, rotational, and vibrational partition functions, $q_{i}^{\mathrm{vtr}}$, the numbers of configurations that the oligomers can adopt are clearly very much different between the fully adsorbed state, the partially detached states, and the transition state to desorption. Furthermore, the configurational partition functions, $q_{i}^{\mathrm{c}}$, are quite temperature dependent. As a result they have a significant impact on the measured values of $\Delta E_{\mathrm{des}}^{*}$.

There are two contributions to the configurational partition function for an oligomer that is partially detached from the surface. The first is the number of ways of detaching $i$ of $I$ segments from the surface. The second contribution arises from the fact that once detached from the surface the segments or $\mathrm{C}-\mathrm{C}$ bonds can adopt three different rotational conformations: trans, +gauche, and - gauche. Together these two contributions give the configurational partition function for the partially detached oligomer

$$
q_{i}^{\mathrm{c}}=\frac{I !}{i !(I-i) !}\left(q^{\mathrm{tg}}\right)^{i}
$$

and thus the overall partition function for a partially detached oligomer.

$$
q_{i}=q_{i}^{\mathrm{vtr}} \frac{I !}{i !(I-i) !}\left(q^{\mathrm{tg}}\right)^{i}
$$

The term $q^{\text {tg }}$ is the partition function for the trans-gauche conformations about each detached bond. One of the assumptions being made here is that in the fully attached state the segments are constrained to the all-trans conformation and thus that for the fully attached alkane the conformational partition function has a value of $q_{0}^{\mathrm{c}}=1$. This is borne out experimentally by STM images which show the alkanes in this conforma- tion when adsorbed on graphite at low temperature. In our previous analysis of this problem, it was assumed that once detached from the surface the segments or $\mathrm{C}-\mathrm{C}$ bonds could adopt all three conformations with equal probability and thus that the partition function for the trans-gauche conformations about each detached segment should have a value of $q^{\mathrm{tg}}=3 .{ }^{20}$ In this paper we have modified our model to recognize that in reality there is an energy difference, $\Delta E_{\mathrm{tg}}^{\mathrm{CC}}$, between the trans and the gauche conformations about each $\mathrm{C}-\mathrm{C}$ bond. For the alkanes the energy difference has been determined by a number of methods which suggest that $\Delta E_{\mathrm{tg}}^{\mathrm{CC}}=2.5 \mathrm{~kJ} / \mathrm{mol}{ }^{23}$ The partition function for the trans-gauche conformations about each detached bond or segment is then given by

$$
q^{\mathrm{tg}}=1+2 \exp \left(\frac{-\Delta E_{\mathrm{tg}}^{\mathrm{CC}}}{k_{\mathrm{B}} T}\right)
$$

The first term is for the ground state trans conformation, and the second is for the two gauche conformations. One of the consequences of this is that the partition function and the entropy for the partially detached oligomers are temperature dependent. Since the value of $\Delta E_{\mathrm{tg}}^{\mathrm{CC}}$ is of the same order of magnitude as $k_{\mathrm{B}} T$, this temperature dependence is significant and is an important correction to the previous model for oligomer desorption. ${ }^{20}$

To describe the equilibrium between oligomers in various stages of partial detachment from the surface we need the free energies, $A_{i}$, which are simply given by

$$
A_{i}=E_{i}-T S_{i}
$$

The energy $E_{i}$ is given by eq 11 above, and the entropy is given by the Boltzmann equation

$$
\mathrm{S}_{i}=k_{\mathrm{B}} \ln \left(q_{i}\right)
$$

where $q_{i}$ is given by eqs 14 and 15 .

\section{Analytic Expression for $\Delta E_{\text {des }}^{\ddagger}$}

Given the expressions for the energies, entropies, and free energies of the oligomers on the surface one can combine eqs 7,9 , and 10 to determine an expression for the measured values of $\Delta E_{\mathrm{des}}^{*}(N)$. The first thing to note is that $\Gamma$, the summation over equilibrium constants, has a simple form.

$$
\begin{aligned}
\Gamma & =\sum_{i=0}^{I} \exp \left(\frac{-\Delta A_{i}}{k_{\mathrm{B}} T}\right) \\
& =\sum_{i=0}^{I} q_{i} \exp \left(\frac{-\Delta E_{i}}{k_{\mathrm{B}} T}\right) \\
& =q^{\mathrm{vtr}} \sum_{i=0}^{I} \frac{I !}{i !(I-i) !}\left(q^{\mathrm{tg}}\right)^{i} \exp \left(\frac{-i \Delta E^{\mathrm{CC}}}{k_{\mathrm{B}} T}\right) \\
& =q^{\mathrm{vtr}}\left(1+q^{\mathrm{tg}} \exp \left(\frac{-\Delta E^{\mathrm{CC}}}{k_{\mathrm{B}} T}\right)\right)^{I}
\end{aligned}
$$

The transition state for desorption is assumed to have all segments detached from the surface, and thus all $I$ segments 
can adopt trans or gauche conformations. The partition function for the transition state is thus

$$
q_{\ddagger}=q_{\ddagger}^{\mathrm{vtr}}\left(q^{\mathrm{tg}}\right)^{I}
$$

where $q_{\ddagger}^{\mathrm{vtr}}$ is the partition function for the degrees of freedom such as translation, vibration, and rotation in the transition state. With these the expression for the desorption rate constant becomes

$$
\begin{array}{r}
k_{\mathrm{des}}=\left(\frac{k_{\mathrm{B}} T}{h}\right) \frac{q_{\ddagger}^{\mathrm{vtr}}}{q^{\mathrm{vtr}}}\left(q^{\mathrm{tg}}\right)^{I}\left(1+q^{\mathrm{tg}} \exp \left(\frac{-\Delta E^{\mathrm{CC}}}{k_{\mathrm{B}} T}\right)\right)^{-I} \times \\
\exp \left(\frac{-\left(I \Delta E^{\mathrm{CC}}+\Delta E\right)}{k_{\mathrm{B}} T}\right)
\end{array}
$$

Finally, eq 9 can be used to derive the expression for the measured value of the $\Delta E_{\mathrm{des}}^{\ddagger}$.

$$
\Delta E_{\mathrm{des}}^{\ddagger}=I\left[\frac{q^{\mathrm{tg}} \Delta E^{\mathrm{CC}}+\left(q^{\mathrm{tg}}-1\right) \Delta E_{\mathrm{tg}}^{\mathrm{CC}}}{q^{\mathrm{tg}}\left(1+q^{\mathrm{tg}} \exp \left(-\Delta E^{\mathrm{CC}} / k_{\mathrm{B}} T\right)\right)}\right]+\Delta E+k_{\mathrm{B}} T
$$

This expression explicitly includes the contributions that arise from the temperature dependence of the configurational partition function $q_{\mathrm{tg}}$. The assumption made in the derivation of this expression is that the partition functions for the nonconformational degrees of freedom in the adsorbed molecule $q^{\mathrm{vtr}}$ and the transition state $q_{\ddagger}^{\text {vtr }}$ roughly cancel and in any case are only weakly temperature dependent. Thus they contribute predominantly to the preexponent for desorption but not to the measured $\Delta E_{\mathrm{des}}^{*}$.

The physical significance of the expression in eq 21 is quite simple although not obvious from the form of the expression. As shown in Appendix A the expression in eq 21 is exactly equivalent to

$$
\Delta E_{\mathrm{des}}^{\ddagger}=\left\langle E^{\ddagger}\right\rangle-\langle E\rangle+k_{\mathrm{B}} T
$$

$\left\langle E^{\ddagger}\right\rangle$ is the average energy of the oligomers in the transition state to desorption and $\langle E\rangle$ is the average energy of the partially detached oligomers adsorbed on the surface. Without the $k_{\mathrm{B}} T$ term, eq 22 is identical to the statistical interpretation of the activation energy for gas-phase reactions given by Fowler and Guggenheim. ${ }^{24}$

\section{Alkane Desorption from Graphite}

This paper applies the analysis above to the desorption of the straight-chain alkanes from the surface of graphite. As has been shown in Figure 1, the measured values of the $\Delta E_{\mathrm{des}}^{*}$ for the alkanes in the length range $\mathrm{C}_{5} \mathrm{H}_{12}$ to $\mathrm{C}_{60} \mathrm{H}_{122}$ are clearly nonlinear in the chain length. To apply the model we think of the alkanes as having only one type of segment: the internal $\mathrm{C}-\mathrm{C}$ bonds. The detachment energy for these segments is given by $\Delta E^{\mathrm{CC}}$. The trans-gauche energy difference for the internal segments is $\Delta E_{\mathrm{tg}}^{\mathrm{CC}}=2.5 \mathrm{~kJ} / \mathrm{mol}$. The expression for the $\Delta E_{\mathrm{des}}^{\ddagger}$ can be fit to the 21 measured values of $\Delta E_{\mathrm{des}}^{+}(N)$ using $\Delta E^{\mathrm{CC}}$ and $\Delta E$ as fitting parameters. Remember that $I=N-1$. Note that the temperatures used in this process were the peak desorption temperatures measured in the TPD experiments and that these increased from $T_{\mathrm{p}}=164 \mathrm{~K}$ for $\mathrm{C}_{5} \mathrm{H}_{12}$ to $T_{\mathrm{p}}=728 \mathrm{~K}$ for the $\mathrm{C}_{60} \mathrm{H}_{122}$. Figure 3 shows that the model fits the

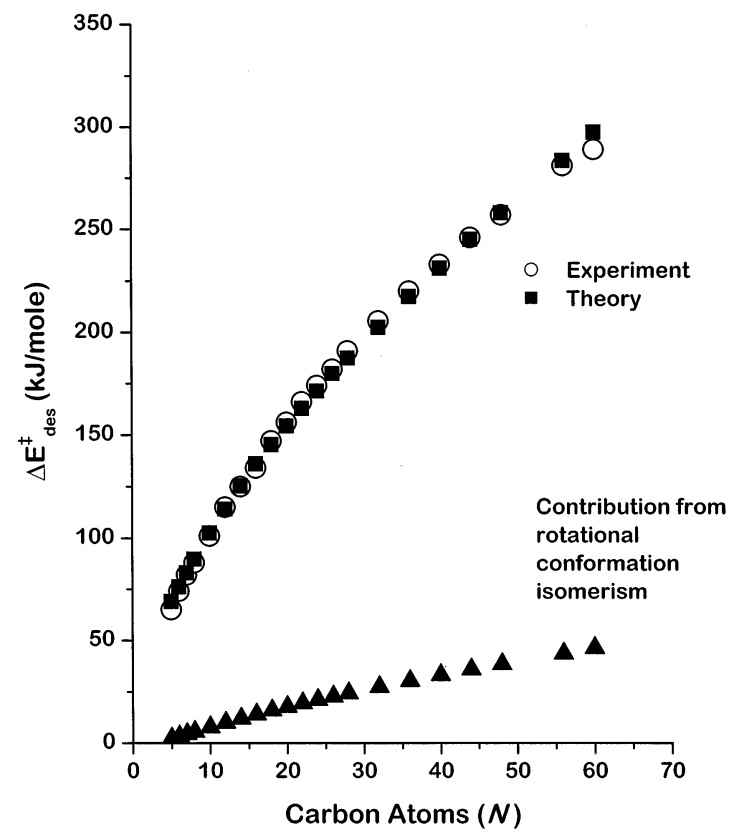

Figure 3. The comparison of the measured values of $\Delta E_{\mathrm{des}}^{\ddagger}$ (open circles: $\bigcirc$ ) for alkane desorption from graphite and the values predicted by our model (solid squares: $\square$ ). The fit is generated by the use of eq 21 with an oligomer detachment energy, $\mathrm{E}_{i}$, that is linear in the number of detached segments (eq 11). The model clearly reproduces the experimental values of $\Delta E_{\mathrm{des}}^{\ddagger}$. The contribution of trans-gauche conformations about each $\mathrm{C}-\mathrm{C}$ bond are illustrated as solid triangles, $\mathbf{\Delta}$, and clearly contribute significantly to the measured values of $\Delta E_{\mathrm{des}}^{\ddagger}$.

experimentally determined values of $\Delta E_{\mathrm{des}}^{*}(N)$ extremely well and yields values of $\Delta E^{\mathrm{CC}}=6.2 \mathrm{~kJ} / \mathrm{mol}$ and $\Delta E=41 \mathrm{~kJ} / \mathrm{mol}$. It is clear that the model reproduces the experiment quite well and the obvious implication is that the configurational isomerism of the alkanes on the graphite surface is leading to the nonlinearity of the $\Delta E_{\mathrm{des}}^{\ddagger}(N)$.

The model used for oligomer desorption described in this paper expands on previous work. ${ }^{20}$ The most important improvement to the model is the derivation of a rigorous, analytical expression for the measured values of $\Delta E_{\mathrm{des}}^{\ddagger}(N)$ and the inclusion of temperature dependence into the partition functions for the trans - gauche conformations about the $\mathrm{C}-\mathrm{C}$ bonds. This latter contribution to the measured values of $\Delta E_{\mathrm{des}}^{\ddagger}(N)$ is given by

$$
\frac{\left(q^{\mathrm{tg}}-1\right) \Delta E_{\mathrm{tg}}^{\mathrm{CC}}}{q^{\mathrm{tg}}\left(1+q^{\mathrm{tg}} \exp \left(-\Delta E^{\mathrm{CC}} / k_{\mathrm{B}} T\right)\right)}
$$

Surprisingly this term makes a significant contribution to the measured values of $\Delta E_{\mathrm{des}}^{+}$. The contribution has been calculated explicitly and is shown in Figure 3. One of the consequences of this inclusion is that the predicted value of the $\Delta E^{\mathrm{CC}}$ drops from the value of $7.8 \mathrm{~kJ} / \mathrm{mol}$ found with $q^{\text {tg }}=3$ to a value of $\Delta E^{\mathrm{CC}}=6.2 \mathrm{~kJ} / \mathrm{mol}$ when using the temperature dependent form for $q^{\text {tg }}$ (eq 15). This value is closer to that measured in studies of shorter chain alcohols and alkanes on a number of metal surfaces. ${ }^{11-18}$

\section{The Origin of $\Delta E$}

We have described the energy of an oligomer on the surface in terms of two quantities: a segment-surface interaction, 
$\Delta E^{\mathrm{CC}}$, whose net contribution to the desorption energy is dependent on the oligomer length and a term $\Delta E$ whose contribution is independent of oligomer length. The physical origin of $\Delta E$ is not clear at this point. Phenomenologically it arises because of the fact that the intercept of experimentally determined values of $\Delta E_{\mathrm{des}}^{\ddagger}(N)$ is nonzero. In other words for short chain lengths the desorption energy can be well approximated by $\Delta E_{\text {des }}^{\ddagger}(N)=\Delta E+b N$. This has been observed in every experimental study of the chain length dependence of alkane desorption energies from surfaces. ${ }^{11-13,15-18}$

The obvious explanation of the physical significance of $\Delta E$ is that it arises from endgroup effects. In other words the segment-surface interaction energy for the $\mathrm{C}-\mathrm{CH}_{3}$ endgroups is not identical to that of the internal $\mathrm{C}-\mathrm{C}$ segments. Since there are only two endgroups, independent of oligomer length, they give rise to a length independent offset to the desorption energies. Unfortunately, this explanation is unlikely to be correct. We have reformulated our model to eliminate $\Delta E$ and instead describe the oligomer-surface interaction in terms of segmentsurface interaction energies arising from the internal segments $\Delta E^{\mathrm{CC}}$ and from two endgroups $\Delta E^{\mathrm{CCH} 3}$. Doing so allows us to fit the data just as well as using eq 21 and gives the same value of $\Delta E^{\mathrm{CC}}=6.2 \mathrm{~kJ} / \mathrm{mol}$ for interaction of the $\mathrm{C}-\mathrm{C}$ segments with the surface. However, it gives a value of $\Delta E^{\mathrm{CCH}_{3}}=26$ $\mathrm{kJ} / \mathrm{mol}$ for the adsorption energy of the $\mathrm{C}-\mathrm{CH}_{3}$ endgroups. It seems physically unreasonable to suggest that the endgroups should have an adsorption energy that is so much higher than that of the internal segments. Furthermore, experiments on the $\mathrm{Cu}(111)$ and $\mathrm{Cu}(100)$ surfaces have shown that the cyclic alkanes $c-\mathrm{C}_{3} \mathrm{H}_{6}$ through $c-\mathrm{C}_{10} \mathrm{H}_{20}$ exhibit the same phenomenological dependence of $\Delta E_{\mathrm{des}}^{\ddagger}(N)$ on length. ${ }^{15,25}$ Although they have no endgroups, the cyclic alkanes exhibit the same nonzero intercept of their desorption energies as the straight-chain alkanes. Clearly, endgroup effects cannot be reasonably invoked as the physical origin of $\Delta E$.

Bishop et al. have suggested that $\Delta E$ arises as a result of an artifact in the way in which the TPD spectra are analyzed to determine the $\Delta E_{\mathrm{des}}^{\ddagger}(N)$. They studied the desorption of alkanes ranging from hexane through decane from the $\mathrm{Pt}(111)$ surface. ${ }^{11}$ On the basis of their observations, they suggest that the presence of islands or other organized structures on the surface can lead to variations in the effective reaction order for desorption and that simply using a first-order equation for all chain lengths will lead to misleading results when measurements are limited to short chain molecules. This is an interesting suggestion but it is a little hard to understand how such effects could persist across the entire range of chain lengths that we have studied on graphite.

A third possibility is that $\Delta E$ some how arises from the fact that the interaction of a segment with the surface depends on its displacement from the surface. We have treated the interaction as though it is a square well potential and segments can be considered to lie either inside or outside the well. This is a common but obviously oversimplified view of the interaction potential. While this is certainly an approximation, it is made for all segments and one would think that it would contribute to the net desorption energy in a manner that is chain length dependent. Thus it is hard to see how it could lead to the length independent term $\Delta E$.

The fact remains that $\Delta E$ appears phenomenologically in all sets of data measuring the desorption energies of homologous series of alkanes from surfaces. ${ }^{11-13,15-18}$ As yet we can find no consistent explanation of its origin. This is a very interesting

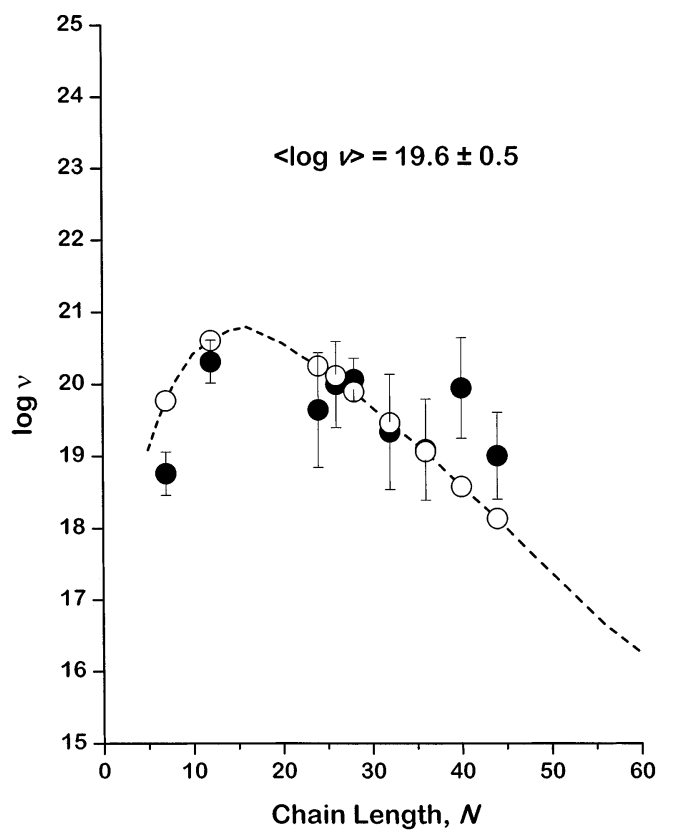

Figure 4. The experimentally determined preexponential factors for alkane desorption from graphite (solid circles: O) determined using variable heating rate desorption methods. ${ }^{20}$ The open circles, $\mathrm{O}$, are the values of the pre-exponents predicted by eq 24 with $q_{\ddagger}^{\mathrm{vtr}} / q^{\mathrm{vtr}}=10^{5}$. The functional form of eq 24 over the range $N=5$ to 60 is shown by the dashed line.

problem but quite distinct from the problem addressed in this paper which is the origin of the nonlinearity of $\Delta E_{\mathrm{des}}^{\ddagger}(N)$.

\section{Desorption Preexponent}

Our study of the desorption of the straight-chain alkanes from the surface of graphite measured both the desorption energies and the pre-exponents to the desorption process, $v \cdot{ }^{20}$ One of the interesting results of that work was the observation that the preexponent for desorption is quite high $(\log v=19.6 \pm 0.5)$ and apparently independent of coverage. The preexponents are shown as solid black circles in Figure 4 and were measured for alkanes with chain lengths of $N=7$ to 44 by using variable heating rate methods to determine both the $\Delta E_{\mathrm{des}}^{\ddagger}$ and $v$ independently. ${ }^{26}$ Our description of the oligomer desorption process ought to be able to predict the chain length dependence of the desorption pre-exponent as well as the $\Delta E_{\mathrm{des}}^{\ddagger}(N)$.

Within the framework of our model for oligomer desorption the desorption pre-exponent is given by

$$
\begin{aligned}
& v=k_{\mathrm{des}} \exp \left(\frac{\Delta E_{\mathrm{des}}^{\ddagger}}{k_{\mathrm{B}} T}\right) \\
&=\left(\frac{k_{\mathrm{B}} T}{h}\right) \frac{q_{\ddagger}^{\mathrm{vtr}}}{q^{\mathrm{vtr}}}\left(q^{\mathrm{tg}}\right)^{I}\left(1+q^{\mathrm{tg}} \exp \left(\frac{-\Delta E^{\mathrm{CC}}}{k_{\mathrm{B}} T}\right)\right)^{-I} \times \\
& \exp \left(\frac{\Delta E_{\mathrm{des}}^{\ddagger}-\left(I \Delta E^{\mathrm{CC}}+\Delta E\right)}{k_{\mathrm{B}} T}\right)
\end{aligned}
$$

All quantities in eq 24 are known except the ratio of the vibrational, translational and rotational partition functions, $q^{\mathrm{vtr}}$. When considering the desorption of small molecules from surfaces, the common assumption is that the ratio of these partition functions is roughly unity leaving a preexponent of $k_{\mathrm{B}} T / h \approx 10^{13} \mathrm{~s}^{-1}$. When measured carefully, however, desorption 
TABLE 1: Fits of Theory to Experiment for Several Different Models of Oligomer Energetics on the Surface

\begin{tabular}{|c|c|c|c|c|c|c|}
\hline \multirow[b]{2}{*}{ model } & \multicolumn{2}{|c|}{ model description } & \multirow[b]{2}{*}{$\Delta E_{\mathrm{des}}^{\ddagger}$ error } & \multirow[b]{2}{*}{$\Delta E$} & \multirow[b]{2}{*}{$\Delta E^{\mathrm{CC}}$} & \multirow[b]{2}{*}{$\Delta E^{\mathrm{CC}-\mathrm{CC}}$} \\
\hline & $E_{i}$ & $\Delta E_{\mathrm{tg}}^{\mathrm{CC}}$ & & & & \\
\hline 1 & $i \Delta E^{\mathrm{CC}}$ & $\Delta E_{\mathrm{tg}}^{\mathrm{CC}}=2.5 \mathrm{~kJ} / \mathrm{mol}$ & $1.6 \%$ & $41 \mathrm{~kJ} / \mathrm{mol}$ & 6.2 & \\
\hline $2^{a}$ & $i \Delta E^{\mathrm{CC}}+(1 / 2) i(i-1) \Delta E^{\mathrm{CC}-\mathrm{CC}}$ & $\Delta E_{\mathrm{tg}}^{\mathrm{CC}}=2.5$ & $0.6 \%$ & 36.5 & 6.8 & 0.013 \\
\hline 3 & $i \Delta E^{\mathrm{CC}}$ & $\begin{array}{l}\Delta E_{\mathrm{tg}}^{\mathrm{CC}}=\infty \\
\left(q^{\mathrm{tg}}=1\right)\end{array}$ & $4.4 \%$ & 53.5 & 5.7 & \\
\hline 4 & $i \Delta E^{\mathrm{CC}}$ & $\begin{array}{l}\Delta E_{\mathrm{tg}}^{\mathrm{CC}}=0 \\
\left(q^{\mathrm{tg}}=3\right)\end{array}$ & $1.2 \%$ & 35 & 7.8 & \\
\hline
\end{tabular}

${ }^{a}$ including the mean field for segment-segment interactions on the surface has required numerical solution for the $\Delta E_{\mathrm{des}}^{\ddagger}(N)$ rather than direct use of eq 21.

preexponents are found to reach values as high as the $10^{19} \mathrm{~s}^{-1}$ observed in this work. This is often attributed to greater translational freedom in the transition state to desorption than in the adsorbed state. ${ }^{27}$ Since we cannot determine the magnitude of the pre-exponent without knowing the value of $q_{\ddagger}^{\mathrm{vtr}} / q^{\mathrm{vtr}}$, we have instead fit the predictions of eq 24 to the experimentally determined values of the pre-exponent by varying $q_{\ddagger}^{\mathrm{vtr}} / q^{\mathrm{vtr}}$. The optimal fit can be achieved using a value of $q_{\ddagger}^{\mathrm{vtr}} / q^{\mathrm{vtr}}=10^{5}$ and then predicts the values of the preexponents shown as open circles in Figure 4.

The first thing to notice from Figure 4 is that the values of $\log v$ found from eq 24 coverage a range of about 2.5 while the experimentally determined values of $\log v$ cover a range of about 1.5. In other words the spread in values over the range of chain lengths examined is roughly similar. The dashed curve in Figure 4 shows the variation in the pre-exponents over chain lengths ranging from $N=5$ to 60 predicted using eq 24 . While the preexponent increases for short chain lengths, it then passes through a maximum and decreases as the chain length is increased further. While the experimental data does not obviously reproduce this behavior, it is not clear that it could be observed easily given the range alkane chain lengths used to determine the pre-exponent. At this point the chain length dependence of the desorption pre-exponent will require further investigation.

\section{Test of Assumptions}

There are a couple of the assumptions of the model for oligomer desorption that can be tested explicitly. This can be done by solving for $\Delta E_{\mathrm{des}}^{\ddagger}(N)$ with different constraints on the model. The results of these tests are listed in Table 1 . The second and third columns list the constraints of the model which are the form of the expression for the energies of the partially detached oligomers and the nature of the conformational partition function $q^{\text {tg }}$ about each detached bond. The fourth column lists the quality of the fit of the predicted values of $\Delta E_{\mathrm{des}}^{\ddagger}(N)$ to the experimental values of $\Delta E_{\mathrm{des}}^{\ddagger}(N)$ quantified as the average fractional difference between the two. Finally, the fifth through seventh columns list the values of the parameters $\Delta E, \Delta E^{\mathrm{CC}}$, and $\Delta E^{\mathrm{CC}-\mathrm{CC}}$ determined from each of these fits. The first row of Table 1 shows the results of the fit of the model described above and illustrated in Figure 3. The average error of the fit is $1.6 \%$ and the resulting estimates of the fitting parameters are $\Delta E=41 \mathrm{~kJ} / \mathrm{mol}$ and $\Delta E^{\mathrm{CC}}=6.2 \mathrm{~kJ} / \mathrm{mol}$.

The first assumption of the model is that the detached segments of the oligomer do not interact with one another and thus that the energy of a partially detached configuration is simply linear in the number of detached segments. This is not expected to be an important issue because the oligomer chain lengths used in this work are all shorter than the persistence length of the alkanes. Nonetheless the interaction between detached segments can be explored by using a mean field description such as eq 25 for the energies $E_{i}$ of the partially detached oligomers.

$$
E_{i}=i \Delta E^{\mathrm{CC}}+(1 / 2) i(i-1) \Delta E^{\mathrm{CC}-\mathrm{CC}}
$$

In this model the unknown parameter $\Delta E^{\mathrm{CC}-\mathrm{CC}}$ is a measure of the strength of interactions between detached segments. The results of the fit to the data are shown in the second row of Table 1 and are obviously improved as a result of the inclusion of a third fitting parameter $\Delta E^{\mathrm{CC}-\mathrm{CC}}$. Note, however, that the predicted values of $\Delta E^{\mathrm{CC}}=6.8 \mathrm{~kJ} / \mathrm{mol}$ and $\Delta E=36.5 \mathrm{~kJ} / \mathrm{mol}$ are not changed significantly. Furthermore, the low value of $\Delta E^{\mathrm{CC}-\mathrm{CC}}=0.013 \mathrm{~kJ} / \mathrm{mol}$ suggests that the inclusion of attractive interactions between detached segments is not a significant factor in the desorption of the oligomers from the surface. The average influence of these interactions for the oligomers with chain lengths in the range $N=5-60$ is roughly $2.6 \%$ of the $\Delta E_{\mathrm{des}}^{\ddagger}$; however, it should be noted that the contributions of segmentsegment interactions increase with increasing chain length and ultimately must become an important contribution to the $\Delta E_{\mathrm{des}}^{\ddagger}$.

It is possible to test the role of the trans-gauche conformations about detached $\mathrm{C}-\mathrm{C}$ bonds in determining the quality of the fit to the experimental values of the $\Delta E_{\mathrm{des}}^{\ddagger}$. Allowing only trans conformations about each $\mathrm{C}-\mathrm{C}$ bond is equivalent to setting the value of $\Delta E_{\mathrm{tg}}^{\mathrm{CC}}=\infty$ in eq 15 or in other words setting $q^{\text {tg }}=1$. These constraints on the model clearly cannot reproduce the observed nonlinearity in $\Delta E_{\text {des }}^{\ddagger}(N)$ and the results are shown in the third row of Table 1. Allowing only trans conformations about detached $\mathrm{C}-\mathrm{C}$ bonds significantly degrades the fit to the experimental results. What is interesting is that the assumption that $\Delta E_{\mathrm{tg}}^{\mathrm{CC}}=0$ or equivalently that there are exactly three energetically equivalent trans-gauche conformations about each $\mathrm{C}-\mathrm{C}$ bond $\left(q^{\text {tg }}=3\right)$ leads to a slight improvement to the fit. The results of the fit using this model are shown in the fourth row of Table 1 and produce a significantly higher value of the $\Delta E^{\mathrm{CC}}=7.8 \mathrm{~kJ} / \mathrm{mol}$. The fact is though that the trans and gauche conformations of the detached bonds cannot be energetically equivalent and so the use of $\Delta E_{\mathrm{tg}}^{\mathrm{CC}}=2.5 \mathrm{~kJ} / \mathrm{mol}$ in the model is favored as it appears to describe the oligomer desorption kinetics very well while incorporating the correct physics.

\section{Configurations of Alkanes Adsorbed on Graphite}

Many of the existing STM images of alkanes adsorbed on the surface of graphite at room temperature show a species 


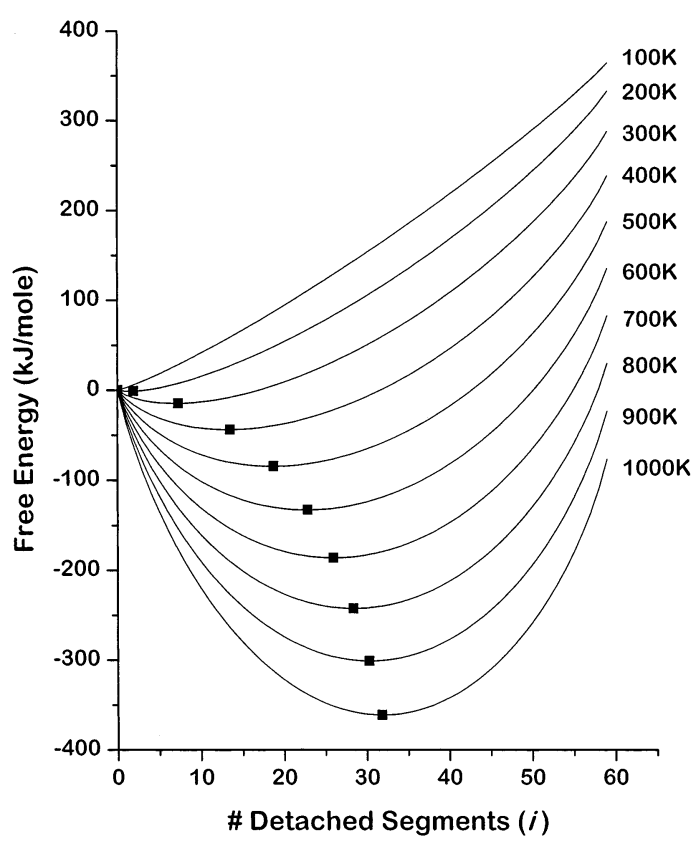

Figure 5. The free energy $A_{i}$ as a function of the number of detached segments, $i$, for $\mathrm{C}_{60} \mathrm{H}_{122}$ adsorbed on graphite. The free energy is given at temperatures ranging from 100 to $1000 \mathrm{~K}$. These free energies are calculated using values of $\Delta E^{\mathrm{CC}}=6.2 \mathrm{~kJ} / \mathrm{mol}$ and $\Delta E=41 \mathrm{~kJ} / \mathrm{mol}$. The average numbers of detached segments at each temperature are indicated by the square data points, $\mathbf{\square}$, and are, of course, close to the values of $i$ which minimize the free energy.

present in an all-trans configuration with the molecular plane lying parallel to the surface. ${ }^{4-10}$ This is the configuration that one would expect from our model at $0 \mathrm{~K}$, in other words under conditions where entropy does not influence the free energies. There is some evidence from the STM images that at higher temperatures the alkane chains undergo some amount of disordering and this is clearly what our model predicts. Of course, the desorption kinetics that serve as the basis for the validity of our model have been able to probe the dynamics of alkane detachment from surfaces over a much wider range of temperatures than has been possible with the STM. The disordering that the model predicts is due to entropic effects which favor the detachment of segments from the surface as the temperature is raised above $0 \mathrm{~K}$. These entropic effects arise from both the large number of ways in which the oligomer can be partially detached from the surface and from the fact that detached segments or bonds can adopt both trans and gauche conformations while segments attached to the surface are restricted to the all-trans configuration. The net result is that the entropic driving force for partial detachment from the surface increases as the chain length increases.

The detachment of oligomer segments from the surface is clearly driven by increasing temperature. Having used our model to determine the values of $\Delta E^{\mathrm{CC}}$ and $\Delta E$ we can predict the nature of the configurations that the molecule will take on the surface at elevated temperatures. Figure 5 shows the free energy for $\mathrm{C}_{60} \mathrm{H}_{122}$ as a function of the number of detached segments, $i$, at temperatures ranging from 100 to $1000 \mathrm{~K}$. The average numbers of detached segments per molecule $\langle i\rangle$ are indicated by the squares in Figure 5 and, of course, are very close to the numbers of detached segments at which the free energies are minimized. The shift in the minimum with increasing temperature indicates that an increasing number of segments are detached from the surface. At $100 \mathrm{~K}$ all segments are attached to the surface. At $300 \mathrm{~K}$ an average of 7.3 segments are detached. Finally at $700 \mathrm{~K}$, just below the desorption temper-

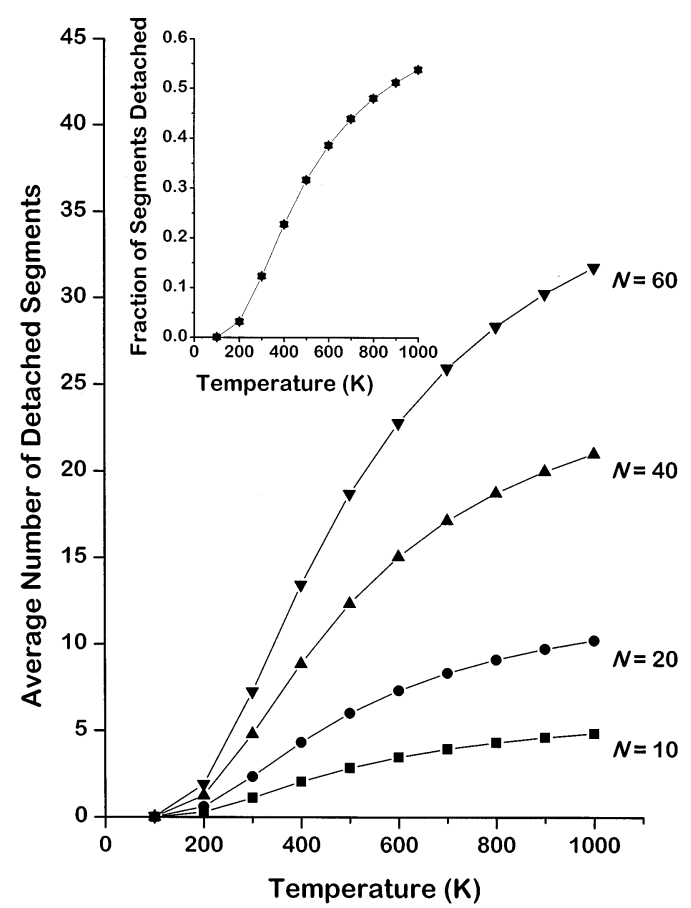

Figure 6. The average numbers of detached segments as a function of temperature for the alkanes with $N=10,20,40$, and 60 carbon atoms on the surface of graphite at temperatures in the range 100$1000 \mathrm{~K}$. The average number of detached segments increases with both chain length and temperature. The inset shows the average fraction of detached segments $\langle i / I\rangle$ as a function of temperature for chains of different length. The average fraction of detached segments is independent of chain length. These were calculated using values of $\Delta E^{\mathrm{CC}}$ $=6.2 \mathrm{~kJ} / \mathrm{mol}$ and $\Delta E=41 \mathrm{~kJ} / \mathrm{mol}$.

ature, an average of 26 segments per molecule are detached from the surface.

As one increases the number of segments in the chain at a given temperature the average number of segments that are detached from the surface increases. This is illustrated in Figure 6 which shows the average numbers of detached segments for chains of length $N=10,20,40$, and 60 carbon atoms over a temperature range from 0 to $1000 \mathrm{~K}$. Clearly, the number of detached segments increases with temperature and with increasing chain length. The inset in Figure 6 shows the fraction of the segments in these molecules that are detached from the surface in the same temperature range. The interesting thing is that the fraction of segments detached at any given temperature is independent of oligomer length. As shown in Appendix B this is a direct result of the statistics describing segment detachment.

Finally, it is important to remember that the number of attached segments of the oligomer at the desorption temperature dictates the dominant term in the expression for the $\Delta E_{\mathrm{des}}^{\ddagger}$. Desorption occurs over a wide range of temperatures for the alkanes used in this work. Figure 7 shows the average numbers of attached and detached segments for the alkanes in the length range $N=5-60$ at their desorption temperatures. As expected, the number of attached segments predicted by our model at the value of the peak desorption temperature, $T_{\mathrm{p}}$, is nonlinear in the chain length. Clearly, however, a large fraction of the segments are detached at the peak desorption temperatures measured in the TPD experiment.

\section{Conclusions}

The dynamics of oligomer desorption from surfaces are clearly influenced by chain length effects in a complex nonlinear 


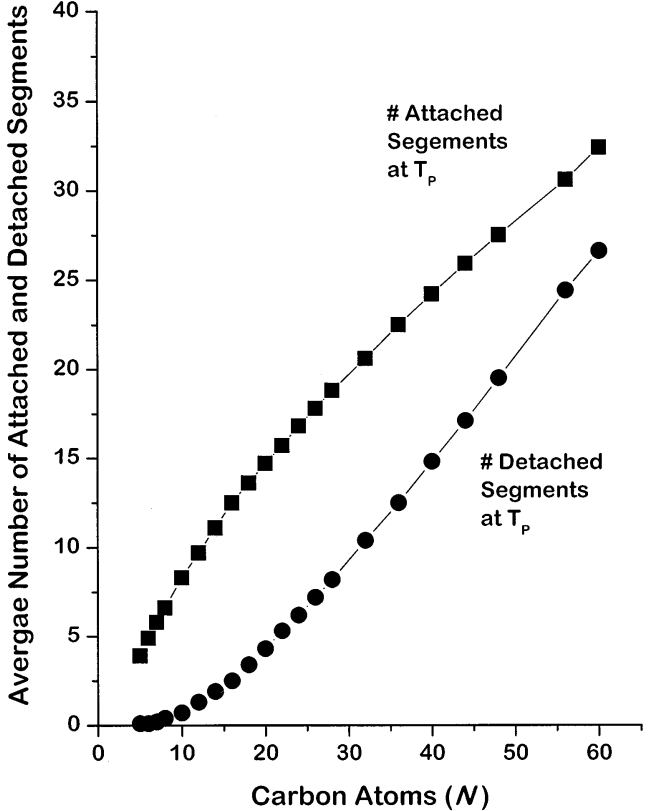

Figure 7. The numbers of attached and detached segments for alkanes on graphite at the peak desorption temperatures measured in the TPD

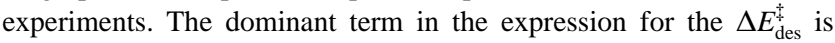
given by the number of attached segments. This number exhibits the same nonlinearity in $N$ as the measured values of $\Delta E_{\mathrm{des}}^{\ddagger}$. These were calculated using values of $\Delta E^{\mathrm{CC}}=6.2 \mathrm{~kJ} / \mathrm{mol}$ and $\Delta E=41 \mathrm{~kJ} / \mathrm{mol}$.

manner. The measured values of $\Delta E_{\mathrm{des}}^{\ddagger}$ in a set of experiments using alkanes in the length range $N=5-60$ have revealed a nonlinear dependence on chain length. An analytical expression for the $\Delta E_{\mathrm{des}}^{\ddagger}$ has been derived that shows that the measured value of $\Delta E_{\mathrm{des}}^{\ddagger}$ is dominated by the difference between the average energy of oligomers in the transition state to desorption and the average energy of the partially detached oligomers on the surface. Ultimately the nonlinearity in the measured values of $\Delta E_{\mathrm{des}}^{\ddagger}$ arises from the fact that configurational isomerism in the adsorbed alkanes favors the existence of partially detached species at temperatures above $0 \mathrm{~K}$.

Acknowledgment. Support from the NSF (CMS-9900647) and from the National Storage Industries Consortium (NSIC) is acknowledged for this work. The development of the high molecular weight doser was supported by the AFOSR (F4962096-1-0253).

\section{Appendix A. Calculation of Average Energies}

The expression in eq 21 for the $\Delta E_{\text {des }}^{\ddagger}(N)$ is consistent with the Fowler-Guggenheim theorem which relates measured values of the activation barriers to the average values of the energies of reactants and transition states. ${ }^{24}$ In this case these are the average energies of the oligomers on the surface and those at the transition state to desorption. Within the framework of our model for oligomer desorption from surfaces the average energy of the oligomers on the surface in their various states of partial detachment is given by

$$
\begin{array}{r}
\langle E\rangle=\frac{1}{Q_{i=0}} \sum_{j=0}^{I}\left(i \Delta E^{\mathrm{CC}}+j \Delta E_{\mathrm{tg}}^{\mathrm{CC}}\right) \frac{I ! \quad j !}{i !(I-i) ! i !(j-i) !} 2^{j} \times \\
\exp \left(\frac{-i \Delta E^{\mathrm{CC}}-j \Delta E_{\mathrm{tg}}^{\mathrm{CC}}}{k_{\mathrm{B}} T}\right)
\end{array}
$$

with the partition function $Q$ given by

$$
\begin{aligned}
Q & =\sum_{i=0}^{I} \sum_{j=0}^{i} \frac{I !}{i !(I-i) !} \cdot \frac{j !}{i !(j-i) !} 2^{j} \exp \left(\frac{-i \Delta E^{\mathrm{CC}}-j \Delta E_{\mathrm{tg}}^{\mathrm{CC}}}{k_{\mathrm{B}} T}\right) \\
& =\sum_{i=0}^{I} \frac{I !}{i !(I-i) !}\left(q^{\mathrm{tg}}\right)^{i} \exp \left(\frac{-i \Delta E^{\mathrm{CC}}}{k_{\mathrm{B}} T}\right) \\
& =\left(1+q^{\mathrm{tg}} \exp \left(\frac{-\Delta E^{\mathrm{CC}}}{k_{\mathrm{B}} T}\right)\right)^{I}
\end{aligned}
$$

The summation over indices $i$ is a summation over the number of detached segments of the oligomer. The summation over $j$ is a summation over the number of gauche conformations among the $i$ detached segments. Note that this partition function is equivalent to $\Gamma$, the sum over equilibrium constants in eqs 7 and 18, except that it includes only configurational terms and excludes contributions from vibrational, rotational, and translational degrees of freedom. The average energy can be expressed analytically as

$\langle E\rangle=\frac{I \exp \left(-\Delta E^{\mathrm{CC}} / k_{\mathrm{B}} T\right)\left[q^{\mathrm{tg}} \Delta E^{\mathrm{CC}}+\left(q^{\mathrm{tg}}-1\right) \Delta E_{\mathrm{tg}}^{\mathrm{CC}}\right]}{1+q^{\mathrm{tg}} \exp \left(-\Delta E^{\mathrm{CC}} / k_{\mathrm{B}} T\right)}$.

In the transition state to desorption the oligomers have all $I$ segments detached from the surface and free to adopt gauche conformations. The average energy is given by

$$
\begin{array}{r}
\left\langle E^{\ddagger}\right\rangle=\frac{1}{Q^{\prime}} \sum_{j=0}^{I}\left(\Delta E+I \Delta E^{\mathrm{CC}}+j \Delta E_{\mathrm{tg}}^{\mathrm{CC}}\right) \frac{I !}{j !(I-j) !} 2^{j} \times \\
\exp \left(\frac{-j \Delta E_{\mathrm{tg}}^{\mathrm{CC}}}{k_{\mathrm{B}} T}\right)
\end{array}
$$

with the partition function given by

$$
\begin{aligned}
Q^{\prime} & =\sum_{j=0 j !(I-j) !}^{I} \frac{I !}{2^{j}} \exp \left(\frac{-j \Delta E_{\mathrm{tg}}^{\mathrm{CC}}}{k_{\mathrm{B}} T}\right) \\
& =\left(1+2 \exp \left(\frac{-\Delta E_{\mathrm{tg}}^{\mathrm{CC}}}{k_{\mathrm{B}} T}\right)\right)^{I} \\
& =\left(q^{\mathrm{tg}}\right)^{I}
\end{aligned}
$$

These give the average energy of the transition state as

$$
\left\langle E^{\ddagger}\right\rangle=\Delta E+I \Delta E^{\mathrm{CC}}+\frac{I\left(q^{\mathrm{tg}}-1\right) \Delta E_{\mathrm{tg}}^{\mathrm{CC}}}{q^{\mathrm{tg}}}
$$

These expressions for the average energies of the reactant (eq 28) and the transition state (eq 31) can be combined through eq 22 to give an expression for $\Delta E_{\mathrm{des}}^{\ddagger}$ which is exactly equal to the expression in eq 21 .

\section{Appendix B. Calculation of the Average Numbers of Detached Segments}

The average number of detached segments for an oligomer of length $I$ is given by the following summation. 


$$
\begin{aligned}
\langle i\rangle & =\frac{1}{Q_{i=0}} \sum_{j=0}^{I} i \frac{I !}{i !(I-i) !} \cdot \frac{j !}{i !(j-i) !} 2^{j} \exp \left(\frac{-i \Delta E^{\mathrm{CC}}-j \Delta E_{\mathrm{tg}}^{\mathrm{CC}}}{k_{\mathrm{B}} \mathrm{T}}\right) \\
& =\frac{1}{Q_{i=0}} \sum_{i}^{I} \frac{I !}{i !(I-i) !}\left(q^{\mathrm{tg}}\right)^{i} \exp \left(\frac{-i \Delta E^{\mathrm{CC}}}{k_{\mathrm{B}} T}\right) \\
& =\frac{1}{Q} I q^{\mathrm{tg}} \exp \left(\frac{-\Delta E^{\mathrm{CC}}}{k_{\mathrm{B}} T}\right)\left(1+q^{\mathrm{tg}} \exp \left(\frac{-\Delta E^{\mathrm{CC}}}{k_{\mathrm{B}} T}\right)\right)^{I-1} \\
& =I \frac{q^{\mathrm{tg}} \exp \left(-\Delta E^{\mathrm{CC}} / k_{\mathrm{B}} T\right)}{\left(1+q^{\mathrm{tg}} \exp \left(-\Delta E^{\mathrm{CC}} / k_{\mathrm{B}} T\right)\right)}
\end{aligned}
$$

The result shows that the average fraction of detached segments $\langle i / I\rangle$ at a given temperature is independent of oligomer chain length as depicted in the inset to Figure 6.

\section{Symbols}

$\Gamma$ : sum over equilibrium constants for oligomer detachment

$\theta_{i}$ : coverage of species with $i$ detached segments

$\theta$ : total coverage of adsorbed oligomers

$A_{i}$ : free energy of partially detached oligomer

$E_{i}$ : energy of oligomer with $i$ segments detached from the surface

$\Delta E_{\mathrm{des}}^{\ddagger}:$ desorption barrier

$\Delta E^{\mathrm{CC}}$ : segment detachment energy for $\mathrm{C}-\mathrm{C}$ bond

$\Delta E_{\mathrm{tg}}^{\mathrm{CC}}$ : trans-gauche energy difference for $\mathrm{C}-\mathrm{C}$ bonds

$\Delta E^{\mathrm{CC}-\mathrm{CC}}$ : energy of interaction between detached segments

$h$ : Planck's constant

$i$ : number of detached segments of an alkane

I: number of segments in alkane $(=N-1)$

$k_{\text {des }}$ : rate constant for desorption

$k_{\mathrm{B}}$ : Boltzmann's constant

$K_{i}$ : equilibrium constant between fully attached oligomers and those with $i$ detached segments

$N$ : number of carbon atoms in alkanes

$q$ : partition function for the adsorbed state

$q_{\ddagger}$ : partition function for the transition state to desorption

$q_{i}$ : partition function for species with $i$ detached segments

$q_{i, \mathrm{c}}$ : conformational partition function for partially detached oligomer $q^{\mathrm{tg}}$ : partition function for trans-gauche conformations about a $\mathrm{C}-\mathrm{C}$ bond

$\mathrm{S}_{i}$ : entropy of partially detached oligomer

$T$ : temperature

$v$ : desorption preexponent

\section{References and Notes}

(1) Tyndall, G. W.; Waltmann, R. J. J. Phys. Chem. B 2000, 104, 7085-7095.

(2) Mate, C. M. Tribol. Lett. 1998, 4, 119-123. 372.

(3) Gellman, A. J. Curr. Opin. Colloid Interface Sci. 1998, 3, 368-

(4) McGonigal, G. C.; Bernhardt, R. H.; Thomson, D. J. Appl. Phys. Lett. 1990, 57, 28-30.

(5) Bucher, J. P.; Roeder, H.; Kern, K. Surf. Sci. 1993, 289, 370-380.

(6) Askadskaya, L.; Rabe, J. P. Phys. Rev. Lett. 1992, 69, 1395-1398.

(7) Rabe, J. P.; Buchholz, S. Makromol. Chem-Macromol. Symp. 1991, 50, 261-268.

(8) Rabe, J. P.; Buchholz, S. Science 1991, 253, 424-427.

(9) McGonigal, G. C.; Bernhardt, R. H.; Yeo, Y. H.; Thomson, D. J. J. Vac. Sci. Technol. B 1991, 9, 1107-1110.

(10) Rabe, J. P.; Buchholz, S. Phys. Rev. Lett. 1991, 66, 2096-2099.

(11) Bishop, A. R.; Girolami, G. S.; Nuzzo, R. G. J. Phys. Chem. B 2000, 104, 754-763.

(12) Wetterer, S. M.; Lavrich, D. J.; Cummings, T.; Bernasek, S. L.; Scoles, G. J. Phys. Chem. B 1998, 102, 9266-9275.

(13) Millot, B.; Methivier, A.; Jobic, H. J. Phys. Chem. B 1998, 102, $3210-3215$.

(14) Zhang, R. M.; Gellman, A. J. J. Phys. Chem. 1991, 95, 74337437.

(15) Teplyakov, A. V.; Gurevich, A. B.; Yang, M. X.; Bent, B. E.; Chen, J. G. G. Surf. Sci. 1998, 396, 340-348.

(16) Slayton, R. M.; Aubuchon, C. M.; Camis, T. L.; Noble, A. R.; Tro,

N. J. J. Phys. Chem. 1995, 99, 2151-2154.

(17) Brand, J. L.; Arena, M. V.; Deckert, A. A.; George, S. M. J. Chem. Phys. 1990, 92, 5136-5143.

(18) Sexton, B. A.; Hughes, A. E. Surf. Sci. 1984, 140, 227-248. (19)

(20) Paserba, K.; Gellman, A. J. J. Chem. Phys. 2001, 115, 6737-6751.

(21) Paserba, K.; Shukla, N.; Gellman, A. J.; Gui, J.; Marchon, B. Langmuir 1999, 15, 1709-1715.

(22) Gellman, A. J.; Paserba, K.; Vaidyanathan, N. Tribol. Lett. 2002, 12, $111-115$.

(23) Smith, G. D.; Jaffe, R. L. J. Phys. Chem. 1996, 100, 18718-18724.

(24) Fowler, H. M.; Guggenheim, E. A. Statistical Thermodynamics; Cambridge University Press: Cambridge, 1939.

(25) Lei, R. Z.; Gellman, A. J. 2002.

(26) Redhead, P. A. Vacuum 1962, 12, 203-211.

(27) Zhdanov, V. P. Surf. Sci. Rep. 1991, 12, 184. 